\title{
Small-Molecules Selectively Modulate Iron-Deficiency Signaling Networks in Arabidopsis
}

\author{
Sakthivel Kailasam, Wei-Fu Chien and Kuo-Chen Yeh* \\ Agricultural Biotechnology Research Center, Academia Sinica, Taipei, Taiwan
}

Plant growth requires optimal levels of iron (Fe). Fe is used for energy production, numerous enzymatic processes, and is indispensable for cellular metabolism. Recent studies have established the mechanism involved in Fe uptake and transport. However, our knowledge of Fe sensing and signaling is limited. Dissecting Fe signaling may be useful for crop improvement by Fe fortification. Here, we report two small-molecules, R3 and R6 [where R denotes repressor of IRON-REGULATED TRANSPORTER 1 (IRT1)], identified through a chemical screening, whose use blocked activation of the Fedeficiency response in Arabidopsis thaliana. Physiological analysis of plants treated with

OPEN ACCESS

Edited by:

Thomas J. Buckhout,

Humboldt-Universität zu Berlin,

Germany

Reviewed by:

Ferenc Fodor

Eötvös Loránd University, Hungary

Rumen Ivanov,

Heinrich-Heine-Universität Düsseldorf,

Germany

*Correspondence:

Kuo-Chen Yeh

kcyeh@gate.sinica.edu.tw

Specialty section:

This article was submitted to Plant Nutrition,

a section of the journal

Frontiers in Plant Science

Received: 17 November 2018

Accepted: 07 January 2019

Published: 31 January 2019

Citation:

Kailasam S, Chien W-F and Yeh K-C (2019) Small-Molecules Selectively Modulate Iron-Deficiency

Signaling Networks in Arabidopsis.

Front. Plant Sci. 10:8.

doi: 10.3389/fp/s.2019.00008
R3 and R6 showed that these small molecules drastically attenuated the plant response to Fe starvation. Small-molecule treatment caused severe chlorosis and strongly reduced chlorophyll levels in plants. Fe content in shoots was decreased considerably by small-molecule treatments especially in Fe deficiency. Small-molecule treatments attenuated the Fe-deficiency-induced expression of the Fe uptake gene IRT1. Analysis of FER-LIKE IRON-DEFICIENCY-INDUCED TRANSCRIPTION FACTOR (FIT) and subgroup Ib basic helix-loop-helix (bHLH) gene (bHLH38/39/100/101) expression showed that R3 affects the FIT-network, whereas R6 affects both the FIT and Ib bHLH networks. An assessment of the effects of the structural analogs of R3 and R6 on the induction of Fe-dependent chlorosis revealed the functional motif of the investigated chemicals. Our findings suggest that small-molecules selectively modulate the distinct signaling routes that operate in response to Fe-deficiency. $R 3$ and $R 6$ likely interrupt the activity of key upstream signaling regulators whose activities are required for the activation of the Fe-starvation transcriptional cascade in Arabidopsis roots.

Keywords: Arabidopsis thaliana, chemical biology, iron deficiency signaling, iron homeostasis, small-molecules

\section{INTRODUCTION}

Many cellular functions occurring during plant growth and development depend on iron $(\mathrm{Fe})$ availability; therefore plants regulate Fe homeostasis by tightly controlling its uptake and allocation. $\mathrm{Fe}$, although abundant in soil, is not so readily available to plants in soils with high $\mathrm{pH}$ due to poor solubility (Colombo et al., 2014). Hence, plants employ different mechanisms for efficient acquisition of Fe from soil. To date, two mechanisms have been identified in higher plants, namely Strategy I or the reduction strategy and Strategy II or the chelation strategy (Kobayashi and Nishizawa, 2012; Connorton et al., 2017) for Fe acquisition. 
Arabidopsis uses Strategy I mode to acquire Fe from soil (Kobayashi and Nishizawa, 2012). For Fe uptake, large amounts of coumarins, facilitated by PLEIOTROPIC DRUG RESISTANCE 9 (PDR9) (Fourcroy et al., 2014; Clemens and Weber, 2016) and protons, mediated by $\mathrm{H}^{+}$-ATPASE 2 (AHA2) (Santi and Schmidt, 2009) are pumped into the rhizosphere. These processes help to solubilize and mobilize the insoluble ferric $\mathrm{Fe}\left(\mathrm{Fe}^{3+}\right)$ in the rhizosphere (Chen et al., 2017; Jeong et al., 2017). Arabidopsis then reduces the soluble $\mathrm{Fe}^{3+}$ into ferrous $\mathrm{Fe}\left(\mathrm{Fe}^{2+}\right)$ by the action of FERRIC REDUCTASE OXIDASE 2 (FRO2) (Robinson et al., 1999) at the cell surface. And the IRON-REGULATED TRANSPORTER 1 (IRT1), a plasma membrane localized divalent cation transporter, then imports ferrous Fe from the extracellular space (Connolly et al., 2002; Vert et al., 2002).

Iron uptake and transport is coordinated by the actions of transcription factors. Several basic helix-loop-helix (bHLH) transcription factors are involved in orchestrating Fe transport and utilization. A subgroup of IIIa bHLH member, FER-LIKE IRON DEFICIENCY-INDUCED TRANSCRIPTION FACTOR (FIT) is involved in controlling the Fe uptake via regulating the expression of IRT1 and FRO2 (Colangelo and Guerinot, 2004; Jakoby et al., 2004; Yuan et al., 2005). Members of subgroup Ib of the bHLH proteins (bHLH38/39/100/101) redundantly interact with FIT and control the Fe uptake-associated genes (Wang et al., 2007, 2013; Yuan et al., 2008). Recent studies have revealed the upstream transcriptional regulation under Fe-starvation. The subgroup IVc bHLH factors (bHLH34/104/105/115), form heterodimers among themselves, directly regulate the expression of $\mathrm{Ib} b H L H$ genes and indirectly regulate the expression of the FIT (Zhang et al., 2015; Li et al., 2016; Liang et al., 2017). The IVc bHLH protein levels are post-translationally controlled by BRUTUS (BTS), a hemerythrin E3 ligase, via proteosomal degradation (Selote et al., 2015). BTS has been proposed to be involved in Fe sensing (Kobayashi and Nishizawa, 2015). BTS negatively regulates the Fe-starvation responses. Hindt et al. showed that the BTS paralogs, BTS LIKE1 (BTSL1) and BTS LIKE2 (BTSL2) act redundantly as negative regulators of the Fe starvation response (Hindt et al., 2017). Therefore, both positive and negative regulators coordinately fine tune the plant responses under the Fe starvation response.

To understand the optimal balance between positive and negative regulation, it is important to shed light on the signaling that is specific to each regulator (positive or negative). By modulating selective signaling branches we might be able to dissect the Fe starvation transcriptional network and the related complicated transcriptional machinery. Many molecules/metabolites such as sucrose, putrescine, nitric oxide (NO) and S-nitrosoglutathione (GSNO), and the hormones auxin and ethylene participate in the signaling process and positively regulate Fe-deficiency transcription (Chen et al., 2010; Lin et al., 2015; Lucena et al., 2015; Liu et al., 2016; Zhu et al., 2016; Kailasam et al., 2018); whereas the hormones, cytokinin, abscisic acid (ABA) and jasmonic acid (JA) act negatively on the network (Liu et al., 2016; Cui et al., 2018). A recent study by Garcia et al. (2018) discussed the different signaling modes, in the form LODIS (LOng Distance Iron Signal) or LODIS-derived and also via NO/GSNO, to the transcription factors. We previously undertook a chemical screening and dissected the Fe-signaling pathway using a small-molecule named R7 (Kailasam et al., 2018). R7 blocked the transfer of the Fe-deficiency generated signal from NO to the FIT by inhibiting the cellular levels of GSNO, a carrier of NO bio-activity, whose levels are critical for the activation of FIT expression. By using the small-molecule R7, we clarified the signaling pathway from NO (Kailasam et al., 2018).

Despite these findings, the identity of the signal that is transferred to transcription factors from NO is still unclear. Moreover, it is not clearly known whether the Fe-dependent signal is conveyed to the transcription factors through only one route or through many routes. With this focus, we used a chemical biology approach to further dissect the signaling routes of Fe starvation response. The chemical screening undertaken yielded two small-molecules named R3 and R6 (R denotes Repressor of IRON-REGULATED TRANSPORTER 1), whose actions during Fe-starvation are uncovered in this report. Smallmolecule treatment resulted in severe Fe-dependent chlorosis and decreased Fe levels in shoots. R6 inhibited the expression of both FIT and $I b b H L H$ genes whereas R3 only inhibited FIT expression. Our finding clearly reveals that these small-molecules modulate Fe-deficiency by targeting specific signaling branches to central transcription factors, further suggesting that multiple routes are used for transferring the Fe-deficiency born signals to the central transcription factors in roots. Our work also highlights that small-molecules can be used to decode novel signaling pathways that modulate the transcription factors responsible for Fe-deficiency.

\section{MATERIALS AND METHODS}

\section{Plant Growth Conditions}

Arabidopsis thaliana Col-0 and the reporter line Pro IRT1:LUC (Kailasam et al., 2018) were used. Seeds were surface-sterilized for $4 \mathrm{~min}$ in $70 \%$ ethanol and treated for $8 \mathrm{~min}$ with $1.2 \%$ sodium hypochlorite containing $0.02 \%$ SDS, finally washed several times in double-distilled $\mathrm{H}_{2} \mathrm{O}$. Two-day-stratified seeds were grown on half-strength Murashige and Skoog ( $1 / 2 \mathrm{MS})$ (Duchefa Biochemie) medium supplemented with $2.3 \mathrm{mM}$ MES, $1 \%$ sucrose and $0.7 \%$ type A agar (Sigma-Aldrich) ( $\mathrm{pH}$ 5.8). For Fe-sufficiency treatments [50 $\mu \mathrm{M} \mathrm{Fe}(\mathrm{II})$-EDTA], $1 / 2 \mathrm{MS}$ was used. For the $\mathrm{Fe} 0$ condition, Fe was omitted [ $1 / 2 \mathrm{MS}$ containing $0 \mu \mathrm{M}$ Fe(II)-EDTA], whereas for the $-\mathrm{Fe}$ condition, $100 \mu \mathrm{M}$ FerroZine was added to the $\mathrm{Fe} 0$ medium. For small molecule treatment, the indicated concentration was added in the medium, whereas in mock treatments dimethyl sulfoxide (DMSO) was added. All plants in this study were grown under a 16-h light/8-h dark photoperiod at $23^{\circ} \mathrm{C}$.

\section{Small Molecule Screening}

The small molecules R3 and R6 were isolated by screening DIVERSet library (ChemBridge, United States) for inhibition of Pro $_{I R T 1}$ :LUC expression (Kailasam et al., 2018). Briefly, the DIVERSet library compounds were dissolved in DMSO and added a final concentration of $100 \mu \mathrm{M}$ to 48 -well plates containing -Fe medium. Two to three $1 / 2 \mathrm{MS}$-grown-seedlings of 
5 day old were transferred to the wells. Two days after treatment, plants were subjected to luminescence analysis. For luminescence assay, plants were submerged in $0.5 \mathrm{mM}$ luciferin solution that contain $0.01 \%$ Triton X-100 and kept for $10 \mathrm{~min}$ in the dark. The luminescence was then captured by using the IVIS Lumina imaging system (Xenogen Corp., United States) with 1-min exposure times.

\section{Protein Isolation and Immunoblot}

Total protein isolation and western blot analysis were conducted according to (Shin et al., 2013). Ten-day-old seedlings underwent a small-molecule treatment for 3 day before analysis. Small molecules were used at a final concentration of $50 \mu \mathrm{M}$. Total protein from roots was extracted by using protein extraction buffer: $125 \mathrm{mM}$ Tris-HCL ( $\mathrm{pH}$ 6.8), 15\% glycerol, 5.5\% SDS, 0.05\% 2-mercaptoethanol, and Protease Inhibitor Cocktail (Roche). SDS-PAGE followed by western-blotting was performed. Blots were probed with an anti-IRT1 antibody (Shanmugam et al., 2011).

\section{Chlorophyll Estimation}

Nine-day-old seedlings that have been grown on $1 / 2 \mathrm{MS}$ media were transferred onto $1 / 2 \mathrm{MS}(\mathrm{Fe} 50)$ or $\mathrm{Fe} 0$ media with 0 or $50 \mu \mathrm{M}$ small molecules. After a 9-day treatment, the leaves were harvested and their fresh weight was measured. Total chlorophyll was extracted in $1.0 \mathrm{ml}$ of $80 \%$ acetone at $4^{\circ} \mathrm{C}$ in the dark for 12-16 h until the leaves became white. The clear supernatant was then analyzed in a spectrophotometer (Power Wave XS; BioTEK) at 470, 646, and $663 \mathrm{~nm}$ spectra. The total chlorophyll content was calculated according to (Wellburn, 1994).

\section{Determination of Elemental Contents}

Tissue elements were estimated by inductively coupled plasmaoptical emission spectrometry (ICP-OES; OPTIMA 5300; PerkinElmer) as described (Shanmugam et al., 2011). Ten-day-old seedlings from $1 / 2 \mathrm{MS}$ plates were transferred to $1 / 2 \mathrm{MS}$ or $\mathrm{Fe} 0$ media containing 0 or $50 \mu \mathrm{M}$ small molecules and grown for 10 days. Shoots were harvested and rinsed with $10 \mathrm{mM} \mathrm{CaCl}_{2}$ for $20 \mathrm{~min}$. After washing with de-ionized water, shoots were dried at $70^{\circ} \mathrm{C}$ for 3 day, then digested with $1 \mathrm{ml} 65 \% \mathrm{HNO}_{3}$ (Merck, Tracepur) and $0.5 \mathrm{ml} \mathrm{H}_{2} \mathrm{O}_{2}$ (Merck, Suprapur). Digested samples were analyzed in ICP-OES for quantification.

\section{Quantitative Reverse Transcription-PCR}

For gene expression analysis, 9-day-old seedlings from $1 / 2 \mathrm{MS}$ plates were transferred to $1 / 2 \mathrm{MS}$ or $-\mathrm{Fe}\left(1 / 2 \mathrm{MS}\right.$ without $\mathrm{Fe}^{2+}$. EDTA and with $100 \mu \mathrm{M}$ FerroZine) containing 0 or $25 \mu \mathrm{M}$ small molecules. After 3 days of treatment, the roots were harvested. RNA isolation, complementary DNA (cDNA) synthesis and quantitative reverse transcription-PCR (qPCR) analysis were conducted according to manufactures protocol. In short, the total RNA was extracted by using Total RNA isolation kit (GeneDireX). The RNA samples were treated with gDNA wipeout RNase-free DNase (Qiagen) at $42^{\circ} \mathrm{C}$ for $2 \mathrm{~min}$ for genomic DNA contamination elimination. Approximately $1 \mu \mathrm{g}$ of total RNA was used for first-strand cDNA synthesis by using a QuantiTect Reverse Transcription kit (Qiagen). 25 ng of RNA was subjected for quantitative PCR (qPCR) using Fast SYBR Green Master Mix (Applied Biosystems) in a 7500 Fast RealTime PCR instrument (Applied Biosystems). Three biological replicates were used for the quantification of expression of each gene. Each biological replicate was analyzed in triplicate. Relative transcript levels were calculated by normalizing to $U B C 21$. Expression was calculated by using the formula $2^{-\Delta C T}$ (Schmittgen and Livak, 2008). The primers described (Zhang et al., 2015) were used for bHLH100 and bHLH101. The primers described in (Shin et al., 2013) were used for IRT1 and UBC21. The primers described in (Shanmugam et al., 2015) were used for FRO2 and FIT. The primers described in (Kailasam et al., 2018) were used for $b H L H 38$ and $b H L H 39$.

\section{Statistical Analysis}

All statistical significance was determined using Student's t test $(P<0.05)$ with SigmaPlot.

\section{RESULTS}

\section{Small Molecules R3 and R6 Block Fe-Deficiency-Induced IRT1 Expression}

We previously employed a chemical screen on Pro IRT1:LUC reporter lines and isolated a small-molecule named R7 ( $\mathrm{R}$ denotes repressor of IRON-REGULATED TRANSPORTER 1) that represses the Fe-deficiency response in Arabidopsis (Kailasam et al., 2018). This screen yielded two more small molecules named R3 and R6. In this report, we analyzed the physiological and molecular responses of plants to understand the role of R3 and R6 in detail. R3 or R6 treatment inhibited the Fedeficiency-inducible Pro $_{I R T 1}$ :LUC expression (Figure 1A). Smallmolecule treatment caused no luminescence in roots under Fedeficient medium as compared to mock-treated that showed stronger luminescence. These results suggest that R3 and R6 may modulate endogenous IRT1 expression.

First, to confirm that the small-molecule effect is not due to dysfunctional Pro IRT1:LUC under the treatment, we analyzed the endogenous IRT1 level under both Fe sufficient and deficient conditions (Figure 1B). As expected, the IRT1 protein was accumulated under Fe deficiency in mock treatment, whereas R3 or R6 treatment abolished the IRT1 accumulation. This indicates that R3 and R6 (Figure 1C) block the accumulation of Fe-deficiency-induced IRT1 protein.

\section{R3 and R6 Cause Severe Fe-Deficiency Chlorosis}

Iron deficiency in the environment causes chlorosis and affects the chlorophyll level in plants. To investigate the effect of small-molecule treatment on plant photosynthetic capacity under Fe starvation, phenotypic analysis was conducted (Figure 2). Compared to mock plants whose leaves were pale-green when grown under Fe-limited conditions, small moleculetreated plants were highly chlorotic (Figure 2A). We further measured the chlorophyll level in both Fe sufficient and deficient 


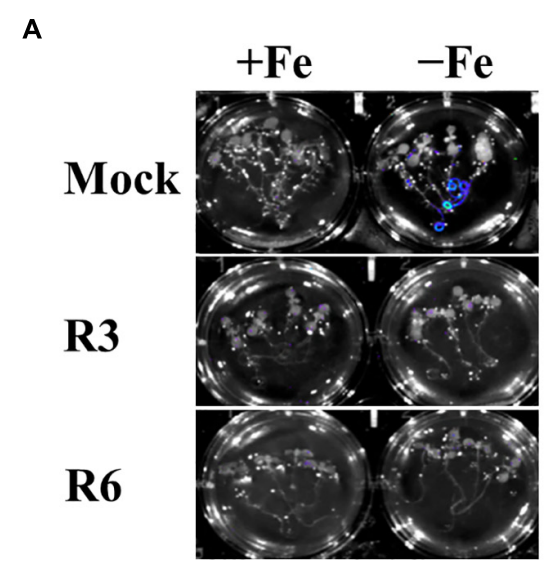

C<smiles></smiles>

R6

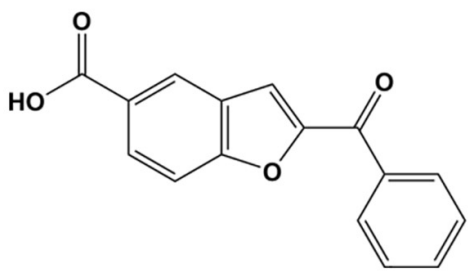

B

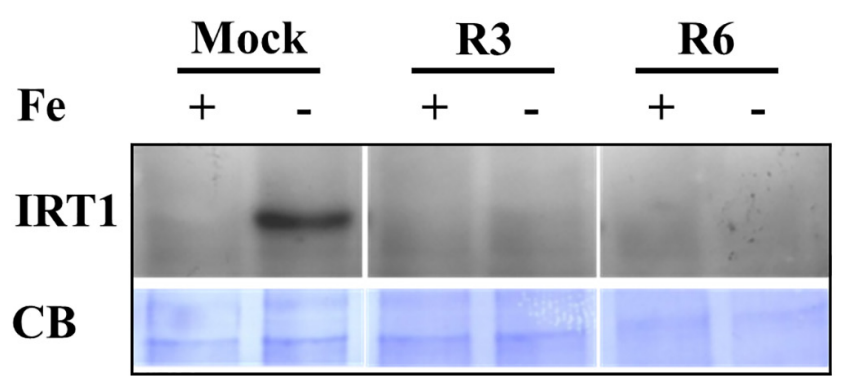

FIGURE 1 | Small-molecules block Fe-deficiency response. (A) Small-molecules inhibit ProlRT 1 :LUC expression. 6-days 1/2MS-grown plants were treated for 3 days under $+\mathrm{Fe}$ or $-\mathrm{Fe}$ media in the presence or absence of $50 \mu \mathrm{M}$ indicated small-molecules. (B) IRT1 protein accumulation in response to small-molecules. The 10-days $1 / 2 \mathrm{MS}$-grown plants were treated for 3 days under $+\mathrm{Fe}$ or $-\mathrm{Fe}$ in the presence or absence of small-molecules. IRT1 protein was detected in total protein extract of roots using an anti-IRT1 antibody. Samples were separated in the same gel. CB, coomassie blue stain. (C) The 2D structure of small-molecules. R3, $\mathrm{N}$-[4-(1,3-benzothiazol-2-yl)-2-methylphenyl] acetamide; R6, 2-benzoyl-1-benzofuran-5-carboxylic acid.

conditions. The small molecule treatment caused a decrease in levels of chlorophyll even under Fe-sufficiency and the levels were drastically reduced under Fe-limited conditions (Figure 2B). These data indicate that R3 and R6 perturb the physiological responses to Fe starvation.

\section{Small-Molecule Treatment Affects Metal Content}

Perturbation in cellular levels of metals often results in chlorosis (Vert et al., 2002). R3 and R6 caused chlorosis; therefore, we next analyzed the cellular level of Fe in response to small molecule treatment (Figure 3). Under Fe sufficiency, R6 treatment did not alter the shoot Fe level, whereas R3 treatment led to a decrease in shoot Fe level (Figure 3A). In Fe-limited medium, the mock treatment showed reduced Fe levels, as expected. The smallmolecule treatment caused a drastic reduction in the levels of shoot Fe under Fe-limited conditions. Since, IRT1 also transports manganese $(\mathrm{Mn})$ and zinc $(\mathrm{Zn})$, we then measured $\mathrm{Mn}$ and $\mathrm{Zn}$ levels. The Mn levels were significantly decreased in response to small molecule treatment under both Fe-sufficiency and deficiency (Figure 3B). R3 or R6 treatment did not alter the $\mathrm{Zn}$ levels in shoots (Figure 3C). These data indicate that R3 and R6 treatment affect cellular metal contents, particularly Fe.

\section{Fe-Acquisition Genes Are Down Regulated in Small-Molecule Treatments}

The above findings suggested that small-molecule treatment might impair the transcription of genes involved in Fe uptake. To test this, we measured the expression levels of Fe-uptakeassociated genes, IRT1, FRO2 and FIT, in response to small molecule treatment in roots. Loss-of-function mutants of these genes display a decrease in cellular Fe levels and chlorosis. We found that IRT1, FRO2, and FIT expression was induced 51.4-, 60.7- and 5.4-fold, respectively by Fe-deficiency in mocktreated plants (Figure 4). The small-molecule treatment strongly inhibited the transcripts of these genes under Fe-deficiency. These results indicate that R3 and R6 inhibit the molecular response to Fe-deficiency by affecting the central transcription factor.

\section{R3 and R6 Are Involved in Different Signaling Branches of Fe-Deficiency}

FIT forms a dimeric complex with members of the Ib bHLH factors (bHLH38/39/100/101) to regulate the expression of Feuptake genes, IRT1 and FRO2. Fe-deficiency also induces the transcripts of $I b b H L H$ genes. Hence, we wondered whether small 
A

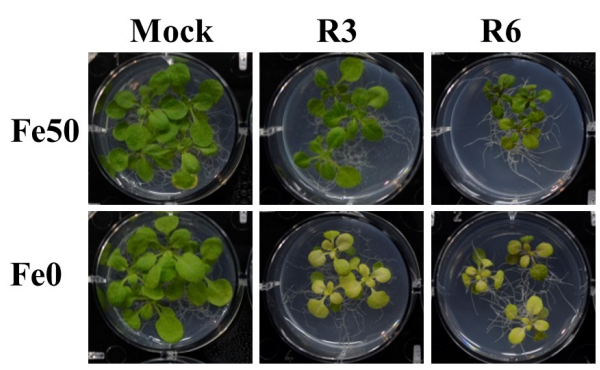

B

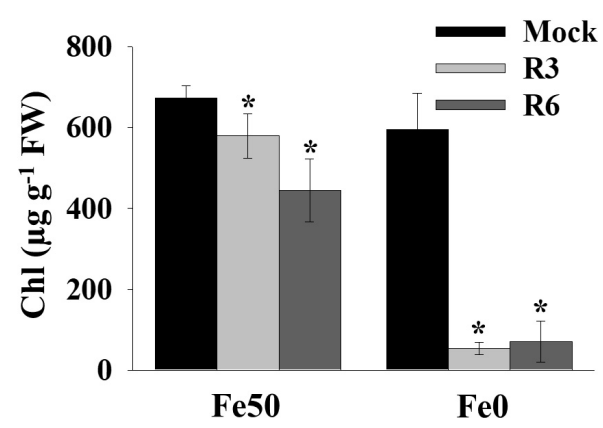

FIGURE 2 | Small-molecules cause severe Fe-deficiency chlorosis. (A) Phenotype of plants with small-molecule treatments. 9-days $1 / 2 \mathrm{MS}$-grown plants were transferred to $\mathrm{Fe} 50$ or $\mathrm{Fe} 0$ media for 9 days in the presence or absence of $50 \mu \mathrm{M}$ indicated small-molecules. (B) Total chlorophyll content of plants under small-molecules treatment. Plants were treated as in (A). Data are mean $\pm S D(n=5)$. *, significant change vs. mock at $P<0.05$ by Student's $t$ test. FW, fresh weight.

molecule treatment deregulates the expression of these genes as well or not. Expression analysis of bHLH38/39/100/101 revealed that their transcripts are indeed induced in mock-treated plants under Fe-deficiency (Figure 5). Interestingly the R3 treatment did not influence the transcript levels of $I b b H L H$ genes under Fe-deficiency whereas R6 treatment inhibited the expression. The R6 inhibition level was $29.4,17.6,41.8$, and $58.0 \%$ of the mock treatment for bHLH38, bHLH39, bHLH100, and bHLH101, respectively. These data indicate that R3 is not involved in the pathway for $I b$ bHLH gene expression whereas R6 is and further implies that small molecules R3 and R6 modulate the Fe-deficiency transcriptional networks selectively.

The reduced expression of Fe-deficiency response transcription factors under small molecule treatment could be the result of defective signaling from plant hormones/metabolites. Auxin, ethylene, NO and GSNO act as positive regulators and exogenous applications of these substances are able to improve plant molecular response and fitness under Fe-starvation. Hence, we were interested in investigating whether providing these substances could alleviate the inhibitory effects caused by R3 or R6. We monitored the Pro IRT1:LUC expression under R3, R6, or R7, a small-molecule that blocks the signal from NO to FIT (Kailasam et al., 2018). LUC expression was not rescued under R3 or R6 treatment by providing any of the positive regulators [naphthaleneacetic acid (NAA) or 1-Aminocyclopropane1-carboxylic acid (ACC) or GSNO] (Figure 6A). Under R7 treatment, supplying NAA or ACC did not rescue the LUC expression either, but supplying GSNO alleviated the R7 inhibition as demonstrated previously (Kailasam et al., 2018). Further we also measured the NO levels under these smallmolecule treatments (Figure 6B). There was sufficient NO level, in fact higher, in roots under Fe deficiency upon treatment with any of the small-molecules. These data suggest that R3 and R6 act independently or downstream of these positive regulators.

\section{Plant Responses to Structural Derivatives of R3 and R6}

Next, to get in-depth insight into the core motif that is required for the action of R3 and R6, structural analogs of the R3 and R6 were searched online in PubChem ${ }^{1}$ and ChemSpider ${ }^{2}$. We randomly selected some of the structural derivatives of R3 and R6 (Table 1), and assayed them. Our results showed that none of the four analogs of R3 assayed had any of the parent activity (Figure 7). They did not produce any observable phenotype under Fe limited conditions. In case of R6, one analog, R6SD1, mimicked the R6 activity; in fact it produced much stronger chlorosis and growth reduction than R6 under both Fe-sufficiency and deficiency (Figure 8). In addition, R6SD1 treatment diminished the IRT1 protein accumulation in roots under Fe-deficiency (Figure $\mathbf{8 C}$ ). The structural analogs, therefore, may help to determine the active motif of the small molecule.

\section{DISCUSSION}

Crop improvement toward fortification of $\mathrm{Fe}$ has great significance for human health as large populations depend on plant-feeds for dietary Fe. Enhancing Fe levels in plants is therefore useful. In order to achieve this, however; an adequate knowledge of Fe homeostasis is needed. Fe homeostasis in plants is controlled through at least five cellular processes: uptake systems, internal transport and distribution, utilization, storage, and finally the regulation (Connorton et al., 2017). Of these coordinated process, uptake is the most critical, that depends on soil $\mathrm{pH}$, redox environment and interactions with other minerals (Colombo et al., 2014). To overcome this kind of environment and for efficient uptake, plants have evolved sophisticated mechanisms. Until now two systems for Fe-uptake, Strategy I, and Strategy II have been identified (Kobayashi and Nishizawa, 2012). Much meticulous work has helped to establish the Fe uptake and transport and the regulation process in Arabidopsis (Brumbarova et al., 2015; Curie and Mari, 2017). However, despite this knowledge, the precise sensing, both external and internal, and the associated signaling for Fe availability is still a poorly understood process.

Small-molecule-based chemical biology is an effective approach to dissect the nutrient-starvation response, especially signaling (Bonnot et al., 2016; Kailasam et al., 2018). In the current study, we investigated the role of two small-molecules,

\footnotetext{
${ }^{1}$ https://pubchem.ncbi.nlm.nih.gov

${ }^{2}$ http://www.chemspider.com
} 

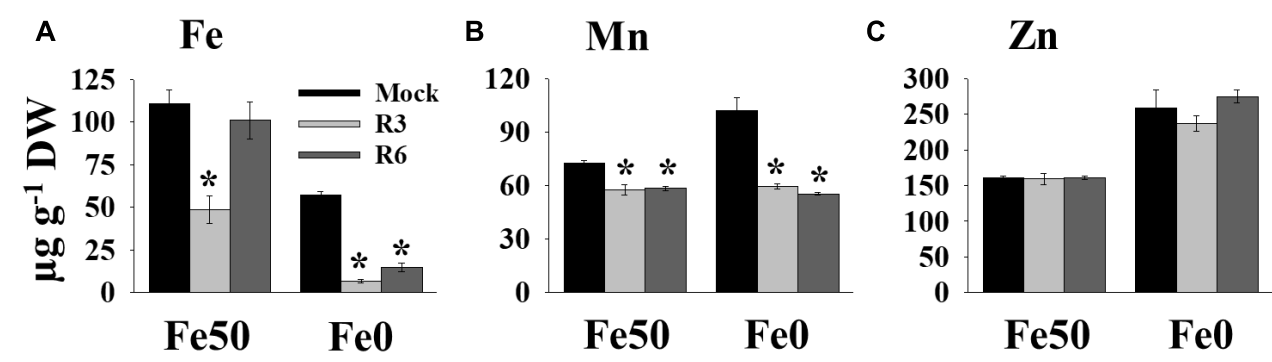

FIGURE 3 | Small-molecules affect Fe levels in plants. Effect of small molecules on Fe (A), Mn (B), and Zn (C) contents in shoots. 10-days $1 / 2$ MS-grown plants were treated with or without $50 \mu \mathrm{M}$ indicated small-molecules under Fe50 or Fe0 condition for 10 days. Levels of elements were measured by ICP-OES. Data are mean $\pm S D(n=3)$. Significant differences compared with mock by Student's $t$ test: ${ }^{*}, P<0.05$. DW, dry weight.
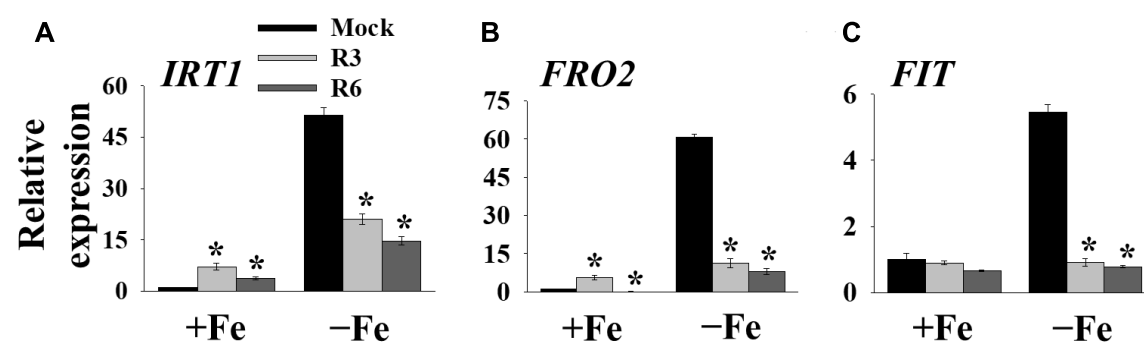

FIGURE 4 | The expression of Fe-acquisition genes is inhibited by small-molecules. qPCR analysis of expression of IRT1 (A), FRO2 (B), and FIT (C) in roots. 9-days $1 / 2 \mathrm{MS}$-grown plants were transferred to +Fe or -Fe in the presence or absence of $25 \mu \mathrm{M}$ indicated small-molecules for 3 days. The expression of UBC21 was used to normalize mRNA levels. The gene expression levels in mock + Fe were set to 1 . Data are mean $\pm S E(n=3)$. Significant differences compared with mock by Student's $t$ test: ${ }^{*}, P<0.05$.

A

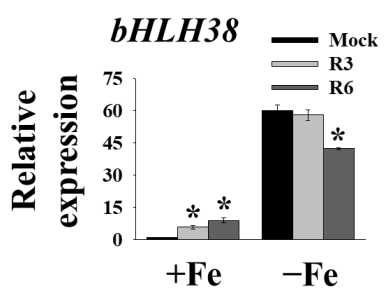

B

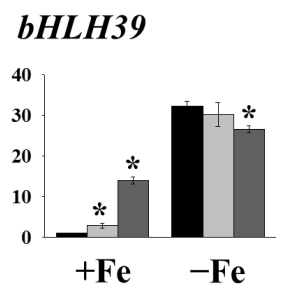

C

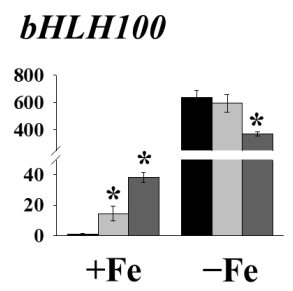

D

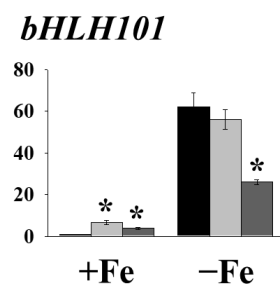

FIGURE 5 | The expression of $I b b H L H$ genes is deregulated by R6 but not by R3. qPCR analysis of expression of $b H L H 38$ (A), $b H L H 39$ (B), $b H L H 100$ (C), and bHLH101 (D) in roots. 9-days $1 / 2 \mathrm{MS}$-grown plants were treated for 3 days under $+\mathrm{Fe}$ or $-\mathrm{Fe}$ in the presence or absence of $25 \mu \mathrm{M}$ indicated small-molecules. The expression of UBC21 was used to normalize mRNA levels. The gene expression levels in mock + Fe were set to 1 . Data are mean $\pm S E(n=3)$. Significant differences vs. mock by Student's $t$ test: ${ }^{*}, P<0.05$.

R3 and R6 in Fe deficiency response (Table 1). R3 and R6 blocked Fe-deficiency induced IRT1 expression (Figure 1). The induction of IRT1 is important for coping with Fe-deficiency (Connolly et al., 2002; Vert et al., 2002). Physiological analysis revealed that R3 and R6 severely affected the chlorophyll levels in plants and imparted stronger chlorosis under Fe-limited conditions (Figure 2). This might be the result of reduced Fe levels under small molecule treatment (Figure 3A). Interestingly, under Fe sufficiency, R3-treated plants had a low Fe level with apparent chlorosis as well. By contrast, R6 treatment reduced the chlorophyll content, with no decrease in shoot Fe level under Fe sufficiency (Figures 2, 3A). Hence, total Fe level is not the only reason for chlorosis. The missing link between Fe content and chlorosis was also observed in the triple mutant bhlh39bhlh100bhlh101, which showed no defective total Fe level in shoots but strong chlorosis (Maurer et al., 2014). Treatment with R3 or R6 affected the Mn level (Figure 3B). Surprisingly the $\mathrm{Zn}$ level, whose level is subjected to increase under Fe-deficiency (Korshunova et al., 1999; Vert et al., 2002), is not affected under both Fe-sufficient and -deficient conditions (Figure 3C). One possibility for the unchanged $\mathrm{Zn}$ levels in shoots is higher translocation rate for $\mathrm{Zn}$. Translocation of $\mathrm{Fe}, \mathrm{Mn}$ and $\mathrm{Zn}$ is depends on the metal-chelating nicotianamine (NA)/citrate levels in the vasculature (Durrett et al., 2007; Schuler et al., 2012). Formation of NA-Zn complexes over Fe and Mn could be favored under 


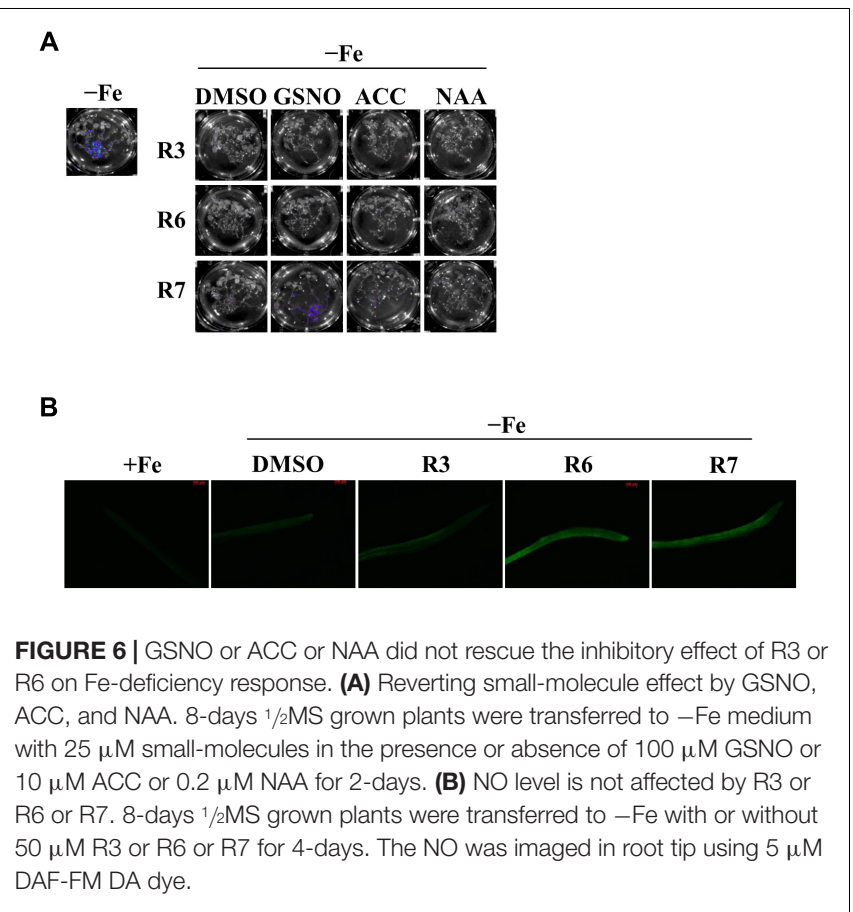

conditions of limited $\mathrm{Fe}$ and the limiting levels of vasculature NA (Palmer et al., 2013). If R3 or R6 treatment brings down the levels of NA, then $\mathrm{Zn}$ would be the favorable substrate for the translocation. Other possibility might account for is that small-molecules may block the transport of metals in a selective manner.

Many genes are strongly induced in response to Fe-deficiency (Buckhout et al., 2009). Both R3 and R6 treatments inhibited the expression of Fe-uptake-associated genes IRT1 and FRO2 (Figures 4A,B). This inhibition is due to low transcript levels of central transcription factor FIT under Fe-deficiency (Figure 4C) upon small molecule treatment. FIT is the central modulator and is responsible for the activation of many Fe-deficiencyassociated genes in root epidermal cells (Mai et al., 2016). We found that R6 downregulated the expression of $\mathrm{Ib} b \mathrm{HLH}$ genes, FIT-partners under Fe-deficiency, whereas R3 did not (Figure 5). Our findings thus reveal that R3 and R6 may target

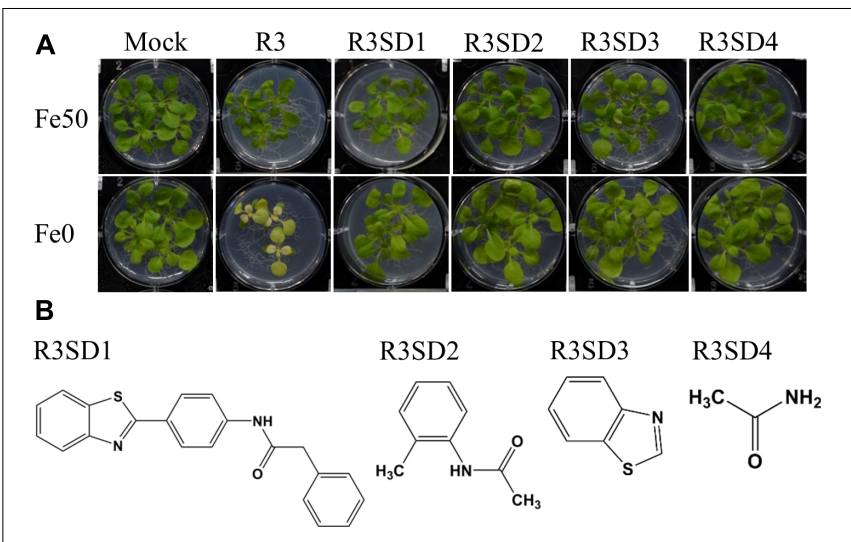

FIGURE 7 | Effect of R3 structural derivatives. (A) The structural derivatives do not mimic the R3 effect. 9-days $1 / 2 \mathrm{MS}$-grown plants were transferred to Fe50 or Fe0 medium for 9 days in the presence or absence of $50 \mu \mathrm{M}$ small-molecules. (B) The 2D structure of R3 structural derivatives.

the transcriptional response through distinct branches under Fe starvation.

The expression of the transcription factors responsible for Fe-deficiency is regulated by the upstream signaling molecules. Any defect in the levels or activity of these signaling-molecules causes decreased expression of transcription factors, and exogenous supply increases the transcription factor expression (Chen et al., 2010; Liu et al., 2016; Kailasam et al., 2018). Based on our data, none of the signaling-molecules (auxin/ethylene/GSNO) alleviated the inhibitory effects caused by R3 or R6 when they were supplied externally (Figure 6). NO levels were higher under R3 or R6 treatment than in the mock (Figure 6C). This supports the notion that R3 and R6 work downstream of auxin/ethylene/NO/GSNO, or alternatively that a novel pathway to the transcription factor exists independent of these hormones (Figures 4-6).

The observed decrease in expression of FIT and $\mathrm{Ib} b \mathrm{HLH}$ transcription factors under R6 treatment (Figures 4, 5) might be due to a blocked signal passage from NO. It has been demonstrated that $\mathrm{NO}$ acts immediately upstream to these transcription factors but downstream of auxin (Chen et al., 2010; Garcia et al., 2010; Kailasam et al., 2018). Recently it has

TABLE 1 | Characteristics of small molecules.

\begin{tabular}{|c|c|c|c|c|c|}
\hline Small molecule & IUPAC name & MW & Molecular formula & ChemSpider ID & PubCem CID \\
\hline R3 & N-[4-(1,3-benzothiazol-2-yl)-2-methylphenyl]acetamide & 282.36 & $\mathrm{C}_{16} \mathrm{H}_{14} \mathrm{~N}_{2} \mathrm{OS}$ & 349964 & 394824 \\
\hline R3SD1 & $\mathrm{N}$-[4-(1,3-benzothiazol-2-yl)phenyl]-2-phenylacetamide & 344.43 & $\mathrm{C}_{21} \mathrm{H}_{16} \mathrm{~N}_{2} \mathrm{OS}$ & 1146425 & 1370084 \\
\hline R3SD2 & $\mathrm{N}$-(2-methylphenyl)acetamide & 149.19 & $\mathrm{C}_{9} \mathrm{H}_{11} \mathrm{NO}$ & 10298354 & - \\
\hline R3SD3 & 1,3-benzothiazole & 135.18 & $\mathrm{C}_{7} \mathrm{H}_{5} \mathrm{NS}$ & 6952 & 7222 \\
\hline R3SD4 & Acetamide & 59.06 & $\mathrm{C}_{2} \mathrm{H}_{5} \mathrm{NO}$ & 173 & 178 \\
\hline R6 & 2-benzoyl-1-benzofuran-5-carboxylic acid & 266.25 & $\mathrm{C}_{16} \mathrm{H}_{10} \mathrm{O}_{4}$ & 6337783 & 8033570 \\
\hline R6SD1 & 1-benzofuran-2-yl(phenyl)methanone & 222.24 & $\mathrm{C}_{15} \mathrm{H}_{10} \mathrm{O}_{2}$ & 21133775 & - \\
\hline R6SD2 & (3-amino-1-benzofuran-2-yl)-phenylmethanone & 237.25 & $\mathrm{C}_{15} \mathrm{H}_{11} \mathrm{NO}_{2}$ & 746595 & 854225 \\
\hline R6SD3 & (3-amino-6-nitro-1-benzofuran-2-yll)-phenylmethanone & 282.25 & $\mathrm{C}_{15} \mathrm{H}_{10} \mathrm{~N}_{2} \mathrm{O}_{4}$ & 4239080 & 5061996 \\
\hline R6SD4 & 1-benzofuran & 118.13 & $\mathrm{C}_{8} \mathrm{H}_{6} \mathrm{O}$ & 8868 & 9223 \\
\hline
\end{tabular}




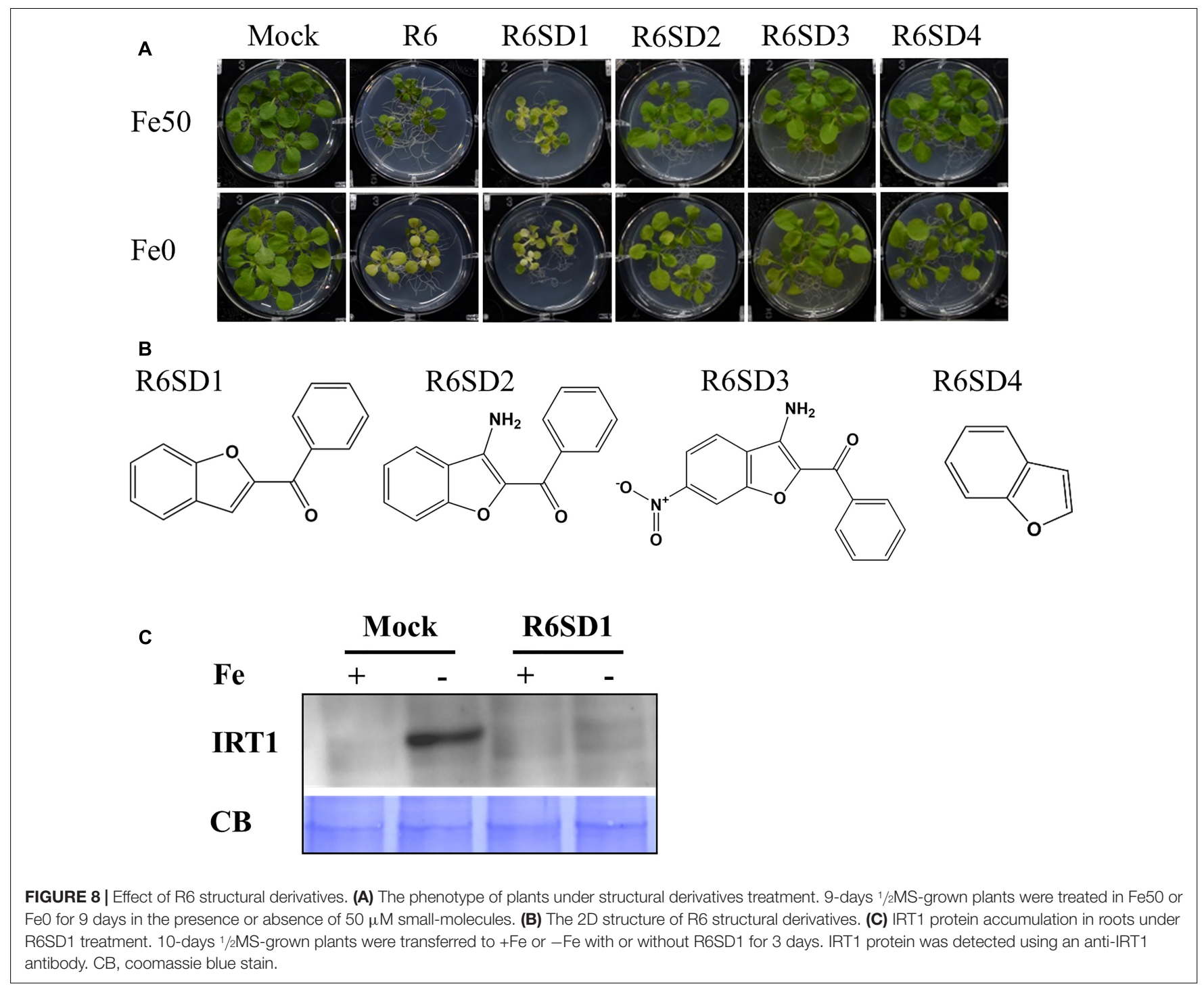

also been shown that IVc bHLH factors (bHLH34/104/105/115) directly control the $I b \quad b H L H$ gene expression and indirectly control FIT (Zhang et al., 2015; Li et al., 2016; Liang et al., 2017). However, it is not clear whether or not IVc bHLH transcription factors work under NO. Our previous study showed that NO did not regulate the transcripts of $I V c b H L H$ genes suggesting that control could be post-translational (Kailasam et al., 2018). One possibility that may account for the effect of R6 is that R6 may target these IVc bHLH proteins thereby reducing the expression of $I b b H L H$ and FIT genes (Figure 9). If this is so, it will be worth investigating how R6 regulates IVc factors.

Given that R3 treatment only affected the expression of FIT and not the $I b$ bHLH transcription factors (Figures 4, 5) and together with the data in Figure 6, it is highly likely that R3 targets the signaling route that is specific to FIT alone (Figure 9). Similar inhibition of the expression of Fe-homeostatic genes was also found with R7 treatment (Kailasam et al., 2018); however, R7 possibly interrupts the signaling pathway from NO to FIT. GSNO has been shown to be involved in mediating the signal from NO specifically to the FIT (Kailasam et al., 2018); however, the precise mechanism and the signal identity is unknown. As the external supply of GSNO did not rescue the R3 inhibition of Pro $_{I R T 1}$ :LUC expression (Figure 6), R3 may interrupt the signal downstream of GSNO or target an independent unknown signaling pathway to FIT. We did not find any structural similarity between R3 and R7. This suggests the presence of multiple signal inputs for FIT, whose routes are selectively and independently targeted by the structurally lessrelated R3 and R7 compounds. Under Fe starvation, a wide range of chemical signals coordinate and trigger the transcriptional response (Liu et al., 2016). Some studies have suggested that cellular Fe, especially the levels in leaf vasculature itself act as a sensing/signaling component (Kumar et al., 2017; Garcia et al., 2018; Khan et al., 2018). Based on these findings, together with action of R3 and R7, it is clear that multilayered signaling networks exist. Importantly, there is lot of interconnection and feed-/forward-back between these signaling molecules, that 


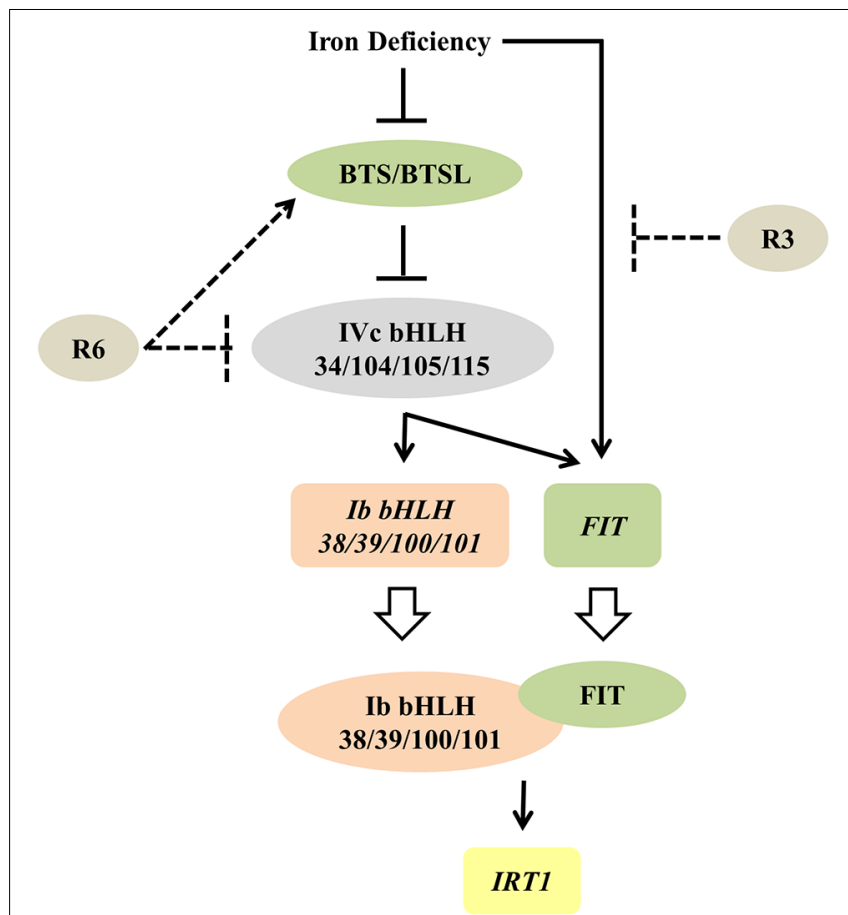

FIGURE 9 | Proposed model for the possible action of R3 and R6 in Fe-deficiency transcriptional-network of Arabidopsis. Fe-status determines the BTS/BTSL stability/function. Under Fe deficiency, BTS is likely degraded, thereby allowing the accumulation of IVc bHLH proteins, which activates the transcription of $I b b H L H$ genes (directly) and FIT (indirectly). R6 inhibits the Fe-deficiency-stimulated expression of these IVc bHLH targets. R6 may block the IVc bHLH function by directly affecting the stability or indirectly promoting the degradation through BTS action. In addition to these signals, an unknown Fe-deficiency born signal directly activates the FIT transcription. FIT forms heterodimer with each member of $\mathrm{lb}$ bHLH transcription factors and regulate the Fe-uptake gene IRT1. R3 most likely intercepts this unknown signal, thus repressing the FIT expression. Solid lines indicate data-supported information from previous studies. Open arrow indicates translation. Proteins are represented by colored-ovals and the genes are indicated by colored-boxes.

influence each other, levels and activity under Fe-starvation (Garcia et al., 2011, 2018; Brumbarova et al., 2015; Liu et al., 2016). Therefore, further study of R3 may reveal the identity of a hidden unknown novel component that regulates the central transcription factor FIT.

Assaying the structural analogs of R3 did not help us to narrow down the active region of $\mathrm{R} 3$ and indicated that modifying the R3 parent compound will lead to loss of activity (Figure 7). R3 belongs to the benzothiazole class of compounds (Figure 1C and Table 1). Benzothiazole derived compounds are used in clinical studies and the benzothiazole moiety has been widely used as a template structure for the development of therapeutic agents (Ali and Siddiqui, 2013). However, the core benzothiazole (R3SD3) structure alone did not mimic the R3 effect and neither did the other R3 derivatives (Figure 7). Therefore, it seems that the parent structure of R3 itself is necessary for its activity. On the other hand, the structural derivatives of R6 provided some clues about the core motif required for the action of R6 (Figure 8). R6 is a benzofuran class compound. Benzofuran is an important pharmacophore and its derivatives are employed in medicinal chemistry for a wide range of drugs (Khanam and Shamsuzzaman, 2015). The benzofuran core (R6SD4) itself did not produce any observable phenotype under Fe-limited conditions. However, one structural derivative R6SD1 mimicked the R6 effect, in fact the phenotype caused more effect than R6 (Figure 8). The main difference between R6 and R6SD1 is that presence of a carboxylic moiety $(-\mathrm{COOH})$ at the fifth position of the benzofuran unit. R6SD1 does not have a $-\mathrm{COOH}$ moiety. It should therefore be worthwhile studying the effect of the $-\mathrm{COOH}$ moiety in $\mathrm{R} 6$ on Fe-deficiency response. Further, characterizing many structural analogs of R3 and R6 might help us to better understand the core motif required for chemical activity, which will in turn benefit identification of its cellular targets, an important study.

\section{CONCLUSION}

The data presented here strongly support the view that small molecules target signaling pathways in the $\mathrm{Fe}$ starvation response network and specifically modulate a particular pathway. This work also shows the usefulness of smallmolecules in dissecting known signal transduction pathway(s). Furthermore, the selective inhibition of signaling pathways suggests the usefulness of R3 and R6 and chemical genetics per se to interpret networks and to identify new components in Fe-signaling. Based on our observations, the smallmolecule R3 targets a novel unknown signaling pathway to the transcription factor FIT, whereas R6 may influence the IVc bHLH transcription factors under Fe-starvation (Figure 9). In summary, this study unraveled a new unknown Fe-signaling route and increases our understanding of plant Fe starvation signaling.

\section{AUTHOR CONTRIBUTIONS}

$\mathrm{K}-\mathrm{CY}$ conceived the research. SK designed and performed the experiments. SK and K-CY wrote the manuscript. W-FC performed the chemical library screening and western-blot. All authors read and approved the manuscript.

\section{FUNDING}

This research was supported by grants (to K-CY) from the Ministry of Science and Technology (MOST 107-2321-B-001018) of Taiwan and Academia Sinica of Taiwan and a postdoc fellowship (to SK) from Ministry of Science and Technology (MOST 107-2811-B-001-578) of Taiwan.

\section{ACKNOWLEDGMENTS}

We thank Jing-Chi Lo and Chia-Lin $\mathrm{Wu}$ for their assistance. 


\section{REFERENCES}

Ali, R., and Siddiqui, N. (2013). Biological aspects of emerging benzothiazoles: a short review. J. Chem. 2013:345198. doi: 10.1155/2013/345198

Bonnot, C., Pinson, B., Clement, M., Bernillon, S., Chiarenza, S., Kanno, S., et al. (2016). A chemical genetic strategy identify the PHOSTIN, a synthetic molecule that triggers phosphate starvation responses in Arabidopsis thaliana. New Phytol. 209, 161-176. doi: 10.1111/nph.13591

Brumbarova, T., Bauer, P., and Ivanov, R. (2015). Molecular mechanisms governing Arabidopsis iron uptake. Trends Plant Sci. 20, 124-133. doi: 10.1016/j.tplants. 2014.11.004

Buckhout, T. J., Yang, T. J., and Schmidt, W. (2009). Early iron-deficiency-induced transcriptional changes in Arabidopsis roots as revealed by microarray analyses. BMC Genomics 10:147. doi: 10.1186/1471-2164-10-147

Chen, W. W., Yang, J. L., Qin, C., Jin, C. W., Mo, J. H., Ye, T., et al. (2010). Nitric oxide acts downstream of auxin to trigger root ferric-chelate reductase activity in response to iron deficiency in Arabidopsis. Plant Physiol. 154, 810-819. doi: 10.1104/pp.110.161109

Chen, Y. T., Wang, Y., and Yeh, K. C. (2017). Role of root exudates in metal acquisition and tolerance. Curr. Opin. Plant Biol. 39, 66-72. doi: 10.1016/j.pbi. 2017.06.004

Clemens, S., and Weber, M. (2016). The essential role of coumarin secretion for Fe acquisition from alkaline soil. Plant Signal. Behav. 11:e1114197. doi: 10.1080/ 15592324.2015.1114197

Colangelo, E. P., and Guerinot, M. L. (2004). The essential basic helix-loophelix protein FIT1 is required for the iron deficiency response. Plant Cell 16, 3400-3412. doi: 10.1105/tpc.104.024315

Colombo, C., Palumbo, G., He, J. Z., Pinton, R., and Cesco, S. (2014). Review on iron availability in soil: interaction of Fe minerals, plants, and microbes. J. Soils Sediments 14, 538-548. doi: 10.1007/s11368-013-0814-z

Connolly, E. L., Fett, J. P., and Guerinot, M. L. (2002). Expression of the IRT1 metal transporter is controlled by metals at the levels of transcript and protein accumulation. Plant Cell 14, 1347-1357. doi: 10.1105/tpc.001263

Connorton, J. M., Balk, J., and Rodriguez-Celma, J. (2017). Iron homeostasis in plants - a brief overview. Metallomics 9, 813-823. doi: 10.1039/c7mt00136c

Cui, Y., Chen, C. L., Cui, M., Zhou, W. J., Wu, H. L., and Ling, H. Q. (2018). Four IVa bHLH transcription factors are novel interactors of FIT and mediate JA inhibition of iron uptake in Arabidopsis. Mol. Plant. 11, 1166-1183. doi: 10.1016/j.molp.2018.06.005

Curie, C., and Mari, S. (2017). New routes for plant iron mining. New Phytol. 214, 521-525. doi: 10.1111/nph.14364

Durrett, T. P., Gassmann, W., and Rogers, E. E. (2007). The FRD3-mediated efflux of citrate into the root vasculature is necessary for efficient iron translocation. Plant Physiol. 144, 197-205. doi: 10.1104/pp.107.097162

Fourcroy, P., Siso-Terraza, P., Sudre, D., Saviron, M., Reyt, G., Gaymard, F., et al. (2014). Involvement of the ABCG37 transporter in secretion of scopoletin and derivatives by Arabidopsis roots in response to iron deficiency. New Phytol. 201, 155-167. doi: 10.1111/Nph.12471

Garcia, M. J., Corpas, F. J., Lucena, C., Alcantara, E., Perez-Vicente, R., Zamarreno, A. M., et al. (2018). A shoot Fe signaling pathway requiring the OPT3 transporter controls GSNO reductase and ethylene in Arabidopsis thaliana roots. Front. Plant Sci. 9:1325. doi: 10.3389/fpls.2018.01325

Garcia, M. J., Lucena, C., Romera, F. J., Alcantara, E., and Perez-Vicente, R. (2010). Ethylene and nitric oxide involvement in the up-regulation of key genes related to iron acquisition and homeostasis in Arabidopsis. J. Exp. Bot. 61, 3885-3899. doi: $10.1093 /$ jxb/erq203

Garcia, M. J., Suarez, V., Romera, F. J., Alcantara, E., and Perez-Vicente, R. (2011). A new model involving ethylene, nitric oxide and Fe to explain the regulation of Fe-acquisition genes in strategy I plants. Plant Physiol. Biochem. 49, 537-544. doi: 10.1016/j.plaphy.2011.01.019

Hindt, M. N., Akmakjian, G. Z., Pivarski, K. L., Punshon, T., Baxter, I., Salt, D. E., et al. (2017). Brutus and its paralogs, BTS LIKE1 and BTS LIKE2, encode important negative regulators of the iron deficiency response in Arabidopsis thaliana. Metallomics 9, 876-890. doi: 10.1039/c7mt00152e

Jakoby, M., Wang, H. Y., Reidt, W., Weisshaar, B., and Bauer, P. (2004). FRU (BHLH029) is required for induction of iron mobilization genes in Arabidopsis thaliana. FEBS Lett. 577, 528-534. doi: 10.1016/j.febslet.2004.10.062
Jeong, J., Merkovich, A., Clyne, M., and Connolly, E. L. (2017). Directing iron transport in dicots: regulation of iron acquisition and translocation. Curr. Opin. Plant Biol. 39, 106-113. doi: 10.1016/j.pbi.2017.06.014

Kailasam, S., Wang, Y., Lo, J. C., Chang, H. F., and Yeh, K. C. (2018). S-Nitrosoglutathione works downstream of nitric oxide to mediate irondeficiency signaling in Arabidopsis. Plant J. 94, 157-168. doi: 10.1111/tpj. 13850

Khan, M. A., Castro-Guerrero, N. A., McInturf, S. A., Nguyen, N. T., Dame, A. N., Wang, J. J., et al. (2018). Changes in iron availability in Arabidopsis are rapidly sensed in the leaf vasculature and impaired sensing leads to opposite transcriptional programs in leaves and roots. Plant Cell Environ. 41, 2263-2276. doi: $10.1111 /$ pce. 13192

Khanam, H., and Shamsuzzaman. (2015). Bioactive benzofuran derivatives: a review. Eur. J. Med. Chem. 97, 483-504. doi: 10.1016/j.ejmech.2014.11.039

Kobayashi, T., and Nishizawa, N. K. (2012). Iron uptake, translocation, and regulation in higher plants. Annu. Rev. Plant Biol. 63, 131-152. doi: 10.1146/ annurev-arplant-042811-105522

Kobayashi, T., and Nishizawa, N. K. (2015). Intracellular iron sensing by the direct binding of iron to regulators. Front. Plant Sci. 6:155. doi: 10.3389/fpls.2015. 00155

Korshunova, Y. O., Eide, D., Clark, W. G., Guerinot, M. L., and Pakrasi, H. B. (1999). The IRT1 protein from Arabidopsis thaliana is a metal transporter with a broad substrate range. Plant Mol. Biol. 40, 37-44. doi: 10.1023/A: 1026438615520

Kumar, R. K., Chu, H. H., Abundis, C., Vasques, K., Rodriguez, D. C., Chia, J. C., et al. (2017). Iron-Nicotianamine transporters are required for proper long distance iron signaling. Plant Physiol. 175, 1254-1268. doi: 10.1104/pp.17. 00821

Li, X. L., Zhang, H. M., Ai, Q., Liang, G., and Yu, D. (2016). Two bHLH transcription factors, bHLH34 and bHLH104, regulate iron homeostasis in Arabidopsis thaliana. Plant Physiol. 170, 2478-2493. doi: 10.1104/pp.15.01827

Liang, G., Zhang, H., Li, X., Ai, Q., and Yu, D. (2017). bHLH transcription factor bHLH115 regulates iron homeostasis in Arabidopsis thaliana. J. Exp. Bot. 68, 1743-1755. doi: 10.1093/jxb/erx043

Lin, X. Y., Ye, Y. Q., Fan, S. K., Jin, C. W., and Zheng, S. J. (2015). Increased sucrose accumulation regulates iron-deficiency responses by promoting auxin signaling in Arabidopsis plants. Plant Physiol. 170, 907-920. doi: 10.1104/pp.15.01598

Liu, X. X., He, X. L., and Jin, C. W. (2016). Roles of chemical signals in regulation of the adaptive responses to iron deficiency. Plant Signal. Behav. 11:e1179418. doi: 10.1080/15592324.2016.1179418

Lucena, C., Romera, F. J., Garcia, M. J., Alcantara, E., and Perez-Vicente, R. (2015). Ethylene participates in the regulation of Fe deficiency responses in strategy I plants and in rice. Front. Plant Sci. 6:1056. doi: 10.3389/fpls.2015.01056

Mai, H. J., Pateyron, S., and Bauer, P. (2016). Iron homeostasis in Arabidopsis thaliana: transcriptomic analyses reveal novel FIT-regulated genes, iron deficiency marker genes and functional gene networks. BMC Plant Biol. 16:211. doi: 10.1186/S12870-016-0899-9

Maurer, F., Arcos, M. A. N., and Bauer, P. (2014). Responses of a triple mutant defective in three iron deficiency-induced BASIC HELIX-LOOP-HELIX genes of the subgroup $\mathrm{Ib}(2)$ to iron deficiency and salicylic acid. PLOS ONE 9:e99234. doi: 10.1371/journal.pone.0099234

Palmer, C. M., Hindt, M. N., Schmidt, H., Clemens, S., and Guerinot, M. L. (2013). MYB10 and MYB72 are required for growth under iron-limiting conditions. PLoS Genet. 9:e1003953. doi: 10.1371/journal.pgen.1003953

Robinson, N. J., Procter, C. M., Connolly, E. L., and Guerinot, M. L. (1999). A ferric-chelate reductase for iron uptake from soils. Nature 397, 694-697. doi: $10.1038 / 17800$

Santi, S., and Schmidt, W. (2009). Dissecting iron deficiency-induced proton extrusion in Arabidopsis roots. New Phytol. 183, 1072-1084. doi: 10.1111/j. 1469-8137.2009.02908.x

Schmittgen, T. D., and Livak, K. J. (2008). Analyzing real-time PCR data by the comparative C(T) method. Nat. Protoc. 3, 1101-1108. doi: 10.1038/nprot. 2008.73

Schuler, M., Rellan-Alvarez, R., Fink-Straube, C., Abadia, J., and Bauer, P. (2012). Nicotianamine functions in the phloem-based transport of iron to sink organs, in pollen development and pollen tube growth in Arabidopsis. Plant Cell 24, 2380-2400. doi: 10.1105/tpc.112.099077 
Selote, D., Samira, R., Matthiadis, A., Gillikin, J. W., and Long, T. A. (2015). Iron-binding $\mathrm{E} 3$ ligase mediates iron response in plants by targeting basic helixloop-helix transcription factors. Plant Physiol. 167, 273-286. doi: 10.1104/pp. 114.250837

Shanmugam, V., Lo, J. C., Wu, C. L., Wang, S. L., Lai, C. C., Connolly, E. L., et al. (2011). Differential expression and regulation of iron-regulated metal transporters in Arabidopsis halleri and Arabidopsis thaliana - the role in zinc tolerance. New Phytol. 190, 125-137. doi: 10.1111/j.1469-8137.2010. 03606.x

Shanmugam, V., Wang, Y. W., Tsednee, M., Karunakaran, K., and Yeh, K. C. (2015). Glutathione plays an essential role in nitric oxide-mediated irondeficiency signaling and iron-deficiency tolerance in Arabidopsis. Plant J. 84, 464-477. doi: 10.1111/tpj.13011

Shin, L. J., Lo, J. C., Chen, G. H., Callis, J., Fu, H., and Yeh, K. C. (2013). IRT1 degradation factor1, a ring E3 ubiquitin ligase, regulates the degradation of iron-regulated transporter1 in Arabidopsis. Plant Cell 25, 3039-3051. doi: $10.1105 /$ tpc.113.115212

Vert, G., Grotz, N., Dedaldechamp, F., Gaymard, F., Guerinot, M. L., Briat, J. F., et al. (2002). IRT1, an Arabidopsis transporter essential for iron uptake from the soil and for plant growth. Plant Cell 14, 1223-1233. doi: 10.1105/tpc. 001388

Wang, H. Y., Klatte, M., Jakoby, M., Baumlein, H., Weisshaar, B., and Bauer, P. (2007). Iron deficiency-mediated stress regulation of four subgroup Ib BHLH genes in Arabidopsis thaliana. Planta 226, 897-908. doi: 10.1007/s00425-0070535-x

Wang, N., Cui, Y., Liu, Y., Fan, H., Du, J., Huang, Z., et al. (2013). Requirement and functional redundancy of Ib subgroup bHLH proteins for iron deficiency responses and uptake in Arabidopsis thaliana. Mol. Plant 6, 503-513. doi: $10.1093 / \mathrm{mp} / \mathrm{sss} 089$
Wellburn, A. R. (1994). The spectral determination of Chlorophyll-a and Chlorophhyll-B, as well as total carotenoids, using various solvents with spectrophotometers of different resolution. J. Plant Physiol. 144, 307-313.

Yuan, Y. X., Wu, H. L., Wang, N., Li, J., Zhao, W. N., Du, J., et al. (2008). FIT interacts with AtbHLH38 and AtbHLH39 in regulating iron uptake gene expression for iron homeostasis in Arabidopsis. Cell Res. 18, 385-397. doi: $10.1038 / C r .2008 .26$

Yuan, Y. X., Zhang, J., Wang, D. W., and Ling, H. Q. (2005). AtbHLH29 of Arabidopsis thaliana is a functional ortholog of tomato FER involved in controlling iron acquisition in strategy I plants. Cell Res. 15, 613-621. doi: 10.1038/sj.cr.7290331

Zhang, J., Liu, B., Li, M., Feng, D., Jin, H., Wang, P., et al. (2015). The bHLH transcription factor bHLH104 interacts with IAA-LEUCINE RESISTANT3 and modulates iron homeostasis in Arabidopsis. Plant Cell 27, 787-805. doi: 10.1105/tpc.114.132704

Zhu, X. F., Wang, B., Song, W. F., Zheng, S. J., and Shen, R. F. (2016). Putrescine alleviates iron deficiency via NO-dependent reutilization of root cell-wall Fe in Arabidopsis. Plant Physiol. 170, 558-567. doi: 10.1104/pp.15.01617

Conflict of Interest Statement: The authors declare that the research was conducted in the absence of any commercial or financial relationships that could be construed as a potential conflict of interest.

Copyright (C) 2019 Kailasam, Chien and Yeh. This is an open-access article distributed under the terms of the Creative Commons Attribution License (CC BY). The use, distribution or reproduction in other forums is permitted, provided the original author(s) and the copyright owner(s) are credited and that the original publication in this journal is cited, in accordance with accepted academic practice. No use, distribution or reproduction is permitted which does not comply with these terms. 